\title{
Spindle assembly in the oocytes of mouse and Drosophila - similar solutions to a problem
}

\author{
Susan Doubilet ${ }^{1} \&$ Kim S. McKim ${ }^{1,2 *}$ \\ ${ }^{1}$ Waksman Institute and Department of Genetics, Rutgers, the State University of New Jersey, Piscataway, NJ, \\ 08854-8020, USA; ${ }^{2}$ Waksman Institute, Rutgers University, 190 Frelinghuysen RD, Piscataway, NJ, 08854, \\ USA; Tel: +1-732-4451164; E-mail: mckim@rci.rutgers.edu \\ *Correspondence
}

Key words: meiosis, acentrosomal, microtubule, central spindle, kinesin-like protein

\begin{abstract}
In the oocytes of many organisms a bipolar spindle is assembled in the absence of centrosomes. In this article we review how this occurs in two model organisms, Drosophila melanogaster and Mus musculus. Common themes include an important role for the chromosomes but paradoxically, organization of a bipolar spindle may not involve kinetochore microtubules. Some comparisons are not yet possible, however, since the same genes have usually not been studied in both systems.
\end{abstract}

\section{Introduction}

In mitosis and meiosis the chromosomes are separated by a machine which includes microtubules and motor proteins. The microtubules make up the mitotic and meiotic spindles and connect the chromosomes to the two poles. The 'classic' model for meiosis I spindle formation, which applies in most male germ cells, begins with the establishment of the two poles, an action that is dependent upon centrosomal-containing microtubule organizing centers (MTOC), each with a pair of centrioles as their focal points (Nicklas 1997) (Figure 1). Astral microtubules, which radiate from the centrosomes and grow and shrink dynamically, make random contacts with the kinetochores. If contacts are made such that both kinetochores of a bivalent become attached to microtubules from opposite poles, they become balanced at the metaphase plate. This condition, known as congression, is maintained by the pull of equal forces in each direction and tension upon the kinetochore-microtubule attachments. If, instead, both kinetochores become attached to the same pole, there is no tension upon the kinetochore-microtubule attachments and the microtubules will become unstable and detach, allowing the kinetochores to make new contacts with microtubules. In this way the chromosomes are active participants in the assembly of the spindle, but only as recipients that capture microtubules growing from the poles (Kirschner \& Mitchison 1986). Essentially the same model applies to mitosis and meiosis II but with sister chromatids instead of bivalents.

But in many species, including Homo sapiens, Caenorhabditis elegans, and Drosophila melanogaster, only the sperm contributes the centrioles to the zygote; the oocyte itself, once it enters meiosis, lacks centrosomes. Thus, meiosis in the oocytes of 

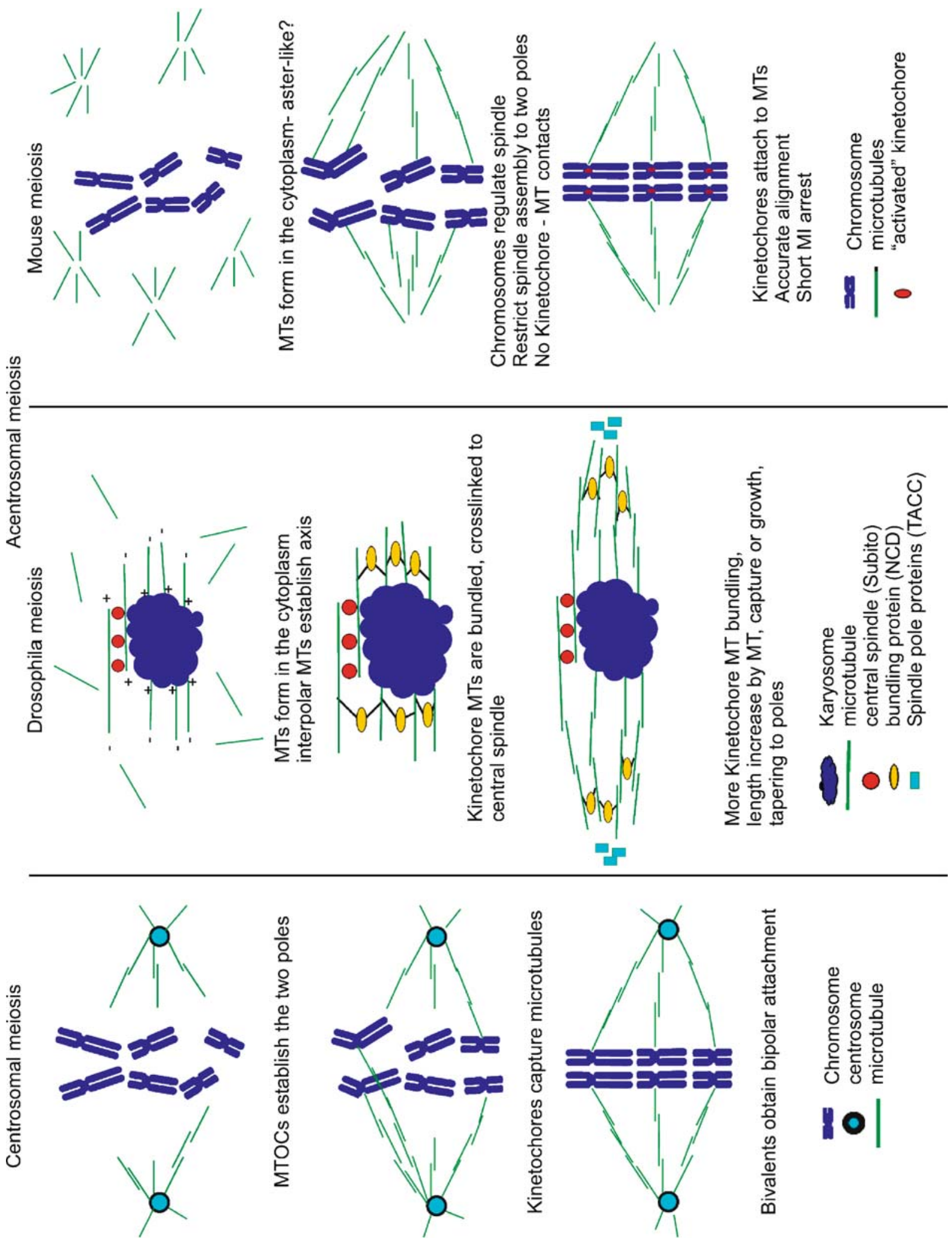

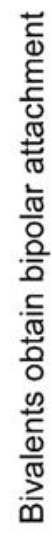

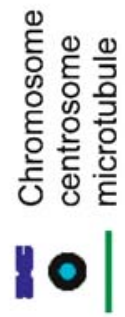


these organisms must take place without MTOCs to guide the assembly of the spindles. This situation gives rise to several questions. For example, how are the microtubules nucleated without centrosomes, and how do they manage to taper to form two poles? Furthermore, how are the locations of the two poles established, so that the homologous chromosomes in meiosis I, and the sister chromatids in meiosis II, are segregated in opposite directions?

The answers to these questions may have important implications toward understanding the differences between males and females in the outcomes of meiosis. Much has been made of the disparity between the sexes in the results of meiosis in humans: the nondisjunction frequency for most chromosomes is far greater in human oocytes than in sperm. Is there a causal relationship between acentrosomal meiosis and a high frequency of nondisjunction and, if so, what is its nature? Several recent reviews have discussed this issue and in particular the 'histories' of the nondisjoining chromosomes. That is, if a pair of chromosomes nondisjoin, did they cross over and, if so, where were the crossovers (Hassold \& Hunt 2001, Morelli \& Cohen 2005, Hall et al. 2006). In this review we compare acentrosomal spindle assembly during meiosis I in the model systems of mouse and fruit fly and investigate how spindle defects could lead to meiosis I nondisjunction.

We will also refer to studies in other organisms, particularly in Xenopus and C. elegans. The study of Xenopus oocytes and their derivative extracts has pioneered models applicable to acentrosomal spindle formation in flies and mammals. For example, a large number of experiments have led to the idea that a combination of motor proteins can assemble microtubules into bipolar spindles in the absence of centrosomes (Compton 1998, 2000, Walczak et al. 1998, Karsenti \& Vernos 2001, Wadsworth \& Khodjakov 2004). This includes motors which are attached to chromosomes and motors which can crosslink microtubules and move toward the minus ends, which could conceivably pull the minus ends together to focus spindle poles.

\section{Drosophila melanogaster}

\section{Overview of oogenesis and assembling an acentrosomal spindle}

Drosophila oocytes develop among 15 nurse cells. Each ancestral germ cell divides four times to produce 16 cystocytes, of which 15 become nurse cells that eventually degenerate. Early in the process, the oocyte enters prophase and completes the DNA repair process that generates crossovers between homologous chromosomes. Then the oocyte pauses at a phase equivalent to diplotene/diakinesis for approximately 3 days during which numerous developmental processes take place (King 1970). Interestingly, if the appropriate experiment is performed, Drosophila oocytes show increased nondisjunction with advancing age (Jeffreys et al. 2003).

Growth and development of the oocyte has been divided into 14 stages (King 1970). During most of this time, corresponding to the prolonged arrest stages of diplotene or diakinesis, the chromosomes are compacted into a spherical mass known as the karyosome. At stage 14 the oocyte's nuclear envelope breaks down and prometaphase I begins with microtubules accumulating in an apolar array around the karyosome. At metaphase I the oocyte arrests again with the spindle parallel to the cell surface and achiasmate homolog sometimes moving precociously toward the poles (Theurkauf \& Hawley 1992). Activation to enter anaphase I is triggered by passage of the oocyte down the oviduct, where the spindle elongates and pivots to become perpendicular to the cell surface (Endow \& Komma 1997, Bloch Qazi et al. 2003). Fertilization of the oocyte triggers meiosis II, during which the two spindles are positioned in tandem. No polar bodies are extruded; the three extra meiotic products remain inside the oocyte and are eventually degraded. Interestingly, orientation of the spindle relative to the cell surface (first parallel then perpendicular) is conserved (McNally \& McNally 2005).

A model for the assembly of the first meiotic spindle (Figure 1), which will be described in detail

Figure 1. Models for spindle assembly with or without centrosomes. In mitotic cells or centrosomal meiosis such as in spermatogonia, microtubules are oriented with their minus ends at the poles and their growing or plus ends toward the chromosomes. This orientation of microtubules is assumed to hold in oocytes, although usually this has not been shown (but see Burbank et al. 2006). The role of interpolar microtubules in mouse acentrosomal spindle assembly has not been investigated. 
in the sections that follow, is based on both fixed (Theurkauf \& Hawley 1992, Jang et al. 2005) and living (Matthies et al. 1996, Skold et al. 2005) oocytes. The organization of the microtubules in early prometaphase I is promoted by the chromosomes, which recruit and/or nucleate the microtubules. As prometaphase I advances, the microtubules are seen to organize around the karyosome into fibers of two forms, the interpolar and kinetochore microtubules. The interpolar microtubules organize tangentially to the karyosome, and then start tapering toward two poles. By metaphase I they run from pole to pole and consist of two sets of fibers that overlap in anti-parallel fashion, the plus ends adjacent to the karyosome and the minus ends directed toward the areas where the two poles will be established. The kinetochore fibers, on the other hand, are organized with their plus ends attached to the kinetochores of the chromosomes. Together, the interpolar microtubules and kinetochore fibers form the meiotic spindle. We will refer to the spindle's middle area, which incorporates the chromosome mass and the overlap area of the interpolar microtubules, as the central spindle.

In the next section we will review the set of proteins that coordinates this process. We will not review other important findings at the periphery of spindle assembly, such as cell-cycle regulators (Pearson et al. 2005, Swan \& Schupbach 2005), regulators of gene expression such as wispy (Brent et al. 2000) or changes in chromosome structure due to histone modifications, all of which appear to be

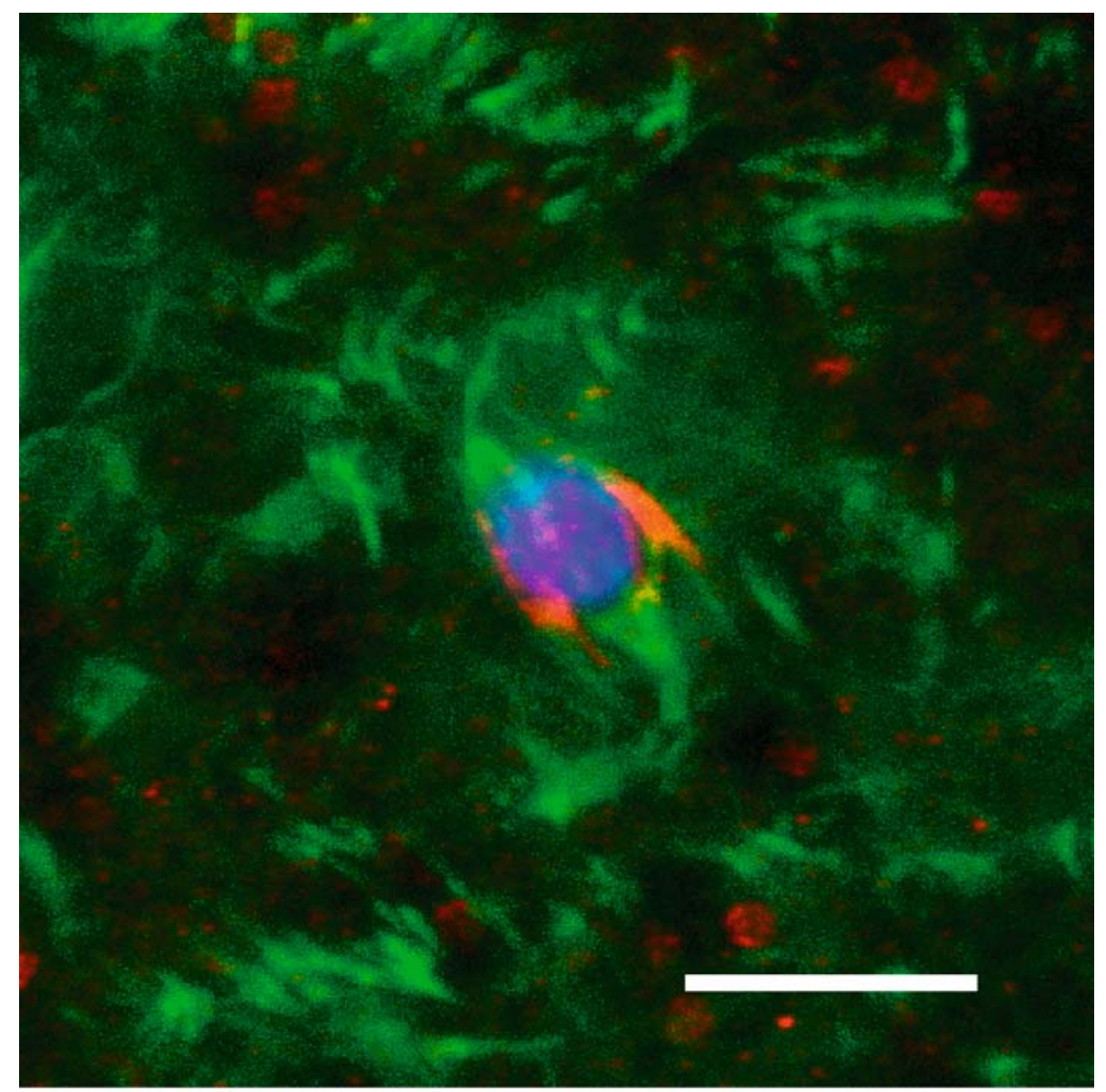

\section{tubulin, DNA, Subito}

Figure 2. A developing spindle in a Drosophila oocyte, with DNA in blue and microtubules in green. Note the abundant cytoplasmic microtubules which may be recruited and bundled together to make the meiotic spindle. The scale bar is $10 \mu \mathrm{m}$. 
important regulators of spindle assembly (Ivanovska et al. 2004, Cullen et al. 2005).

\section{Chromosomes recruit or nucleate microtubules, stimulating spindle formation}

Unlike in mitotic cells, astral MTOCs are not observed before nuclear envelope breakdown (NEB) and $\gamma$-tubulin, CP-60 and CP-190, typical at mitotic MTOCs, are not found at meiotic spindle poles (Theurkauf \& Hawley 1992, Matthies et al. 1996). Thus, the action of the poles in microtubule nucleation and establishing the axis of division does not apply in acentrosomal meiosis. Instead, it has been shown that the chromosomes can take an active role. D. melanogaster chromosomes are capable of capturing free microtubules present in the cytoplasm (Figure 2), and it is this action which may trigger spindle formation (Theurkauf \& Hawley 1992, Hatsumi \& Endow 1992, McKim \& Hawley 1995). There is currently no evidence that the chromosomes nucleate microtubule fiber growth (see 'Summary and the future'). Even a single, small chromosome, like the D. melanogaster fourth chromosome, has been shown to stimulate this action, and similar activities have been observed in Xenopus extracts (Karsenti et al. 1984a,b).

The chromosomes also have a role in maintaining the metaphase I arrest. In the absence of crossovers no chiasmata form. Presumably due to the lack of tension, the chromosomes move precociously to the poles (McKim et al. 1993, Jang et al. 1995). If there is at least one chiasma, the resulting tension is enough to keep the oocyte in metaphase. This is opposite to the effect in a mitotic cell, where lack of tension signals a delay in anaphase onset.

The central spindle as an organizer of acentrosomal meiosis

The meiotic metaphase central spindle incorporates the karyosome and the overlap area of the interpolar microtubules and is related to the mitotic midzone by its constituent proteins. It has been identified by a bright band of tubulin staining which is particularly intense due to overlapping antiparallel microtubule fibers (Theurkauf \& Hawley 1992, McKim et al. 1993). In addition, this bright band of tubulin staining colocalizes with many (but not all, such as Polo) of the proteins found in the mitotic midzone at anaphase (Jang et al. 2005). These include RacGap50C, the motor proteins Klp61F, Pavarotti and Subito as well as the Passenger proteins Aurora B and Incenp. Many of these proteins have an important role in cytokinesis (e.g. Giansanti et al. 1998, D'Avino et al. 2005) but except for Subito (see below), their role in meiosis is not known.

A striking feature of the meiotic central spindle is its early emergence. In the Drosophila oocyte the meiotic central spindle forms at the earliest stages of prometaphase, immediately after NEB, when microtubules are assembled around the chromosomes but the poles have not yet been established (Jang et al. 2005). Many of the midzone proteins listed above are found on the central spindle during meiotic prometaphase I. In mitosis these proteins normally do not appear on the spindle until mitotic anaphase. For example, the Passenger proteins move from the mitotic centromeres to the midzone at anaphase whereas in acentrosomal meiosis they do not appear at the centromeres. This early formation of the central spindle may be an important adaptation that compensates for the absence of centrosomes, as the central spindle may play an important function in establishing the orientation of the spindle poles (Jang et al. 2005).

Another function for the central spindle is suggested from studies of the centrosomal protein centrosomin $(\mathrm{CNN})$, which is required for centrosome function in mitotic cells. In contrast to the centrosome localization in mitotic cells, $\mathrm{CNN}$ is present at the meiotic central spindle during meiosis I (Riparbelli \& Callaini 2005). Cnn mutants appear to have no defects during meiosis I, but fail to form a properly developed central MTOC in meiosis II. Late in anaphase I the localization of $\mathrm{CNN}$ to the central spindle is joined by ASP, which usually is present at the poles (see below). It is possible that CNN localization to the central spindle is in preparation for meiosis II (Riparbelli \& Callaini 2005).

\section{Central spindle formation requires Subito}

Subito (SUB) is the Drosophila ortholog of human mitotic kinesin-like protein 2 (MKLP2), a plusend-directed motor (Giunta et al. 2002) in the kinesin- 6 family. At the onset of meiotic prometaphase, SUB localizes to the central spindle adjacent to the karyosome. Uni- and tri-polar spindles are frequent in null and hypomorphic mutants of sub (Giunta 
et al. 2002), indicating that, while SUB is associated with the orientation and stabilization of precisely two poles, it is not essential for the formation of the poles themselves. SUB is required for development or maintenance of the central spindle, which is evidence that the central spindle has an important role in the formation of the bipolar spindle (Jang et al. 2005). This role for SUB could be related to an activity bundling antiparallel microtubules (Nislow et al. 1992), the type which make up the central spindle. In the absence of SUB, the central spindle is absent and the correct number of spindle poles does not form. Furthermore, SUB is required for the localization of the Passenger proteins Aurora B and Incenp in mitosis and meiosis, the very recruitment of which may stimulate chromosome-microtubule interactions and thus, in turn, encourage central spindle formation.

\section{Kinetochore fiber organization and the bundling of microtubules by NCD to taper the poles}

As microtubules become organized after NEB, those that will become the kinetochore fibers attach themselves at their plus ends to the karyosome. It is not known whether this occurs at about the same time as the initiation of the central spindle or shortly thereafter. The importance of bundling microtubules has been revealed by the analysis of NCD (non-claret disjunctional), a minus-end-directed kinesin-14 motor protein with microtubule bundling properties (McDonald et al. 1990, Chandra et al. 1993). While bipolar spindles can be formed in the absence of NCD, they occur with far less efficiency and stability than when NCD is present (Matthies et al. 1996, Skold et al. 2005). The assembly of a morphologically normal bipolar spindle takes considerably longer in ncd mutant oocytes than in wild-type, normally 20-25 min. Furthermore, in ncd mutant oocytes, if a bipolar spindle does form it will often degenerate and then re-form again, whereas wildtype spindles, once formed, are very stable structures.

The phenotypes of $n c d$ mutants may result from two roles of NCD in spindle assembly. First, NCD has a role in the initial recruitment of microtubules around the karyosome. 'Lateral associations' between microtubules and the chromosomes that depend on NCD have been observed in live oocyte studies (Skold et al. 2005). This activity could contribute to central spindle formation and may not be important for the formation of kinetochore microtubules. Thus, like Subito, NCD may have a role in the initial kinetochore-independent recruitment of microtubules around the karyosome.

Second, Matthies et al. (1996) have developed a model for an NCD-dependent tapering of the spindle poles. According to this model, NCD binds to the microtubules at their points of association with the chromatin; that is, at the microtubule plus ends. This is believed to occur soon after NEB, once the cytoplasmic microtubules have associated with chromatin. Then, NCD moves toward the minus ends, bridging between the microtubule fibers and bundling them together. This action, it is believed, first brings the fibers into parallel alignment and then clusters them at their minus ends, thereby generating the poles. For both the recruitment and tapering functions, NCD may be partially redundant with other motor proteins. The activity of another motor, likely dynein, has been proposed because, although spindle pole formation is abnormal in a null ncd mutant, spindle poles do eventually form. The contribution of minus-end-directed motors, like NCD and dynein, to acentrosomal spindle assembly has also been described in Xenopus extracts (Walczak et al. 1998) and Drosophila S2 cells (Goshima et al. 2005).

\section{Importance of the meiotic central spindle; communication between the interpolar and kinetochore microtubules}

The activity of minus end directed motors explains the focusing of the poles for each 'half spindle', but how is assembly of the two half spindles coordinated? The central spindle may form the 'backbone' structure that defines the orientation of the spindle axis (Jang et al. 2005). The central spindle must then in some way 'communicate' with the kinetochore fibers so that they become aligned parallel to this axis. This communication may occur by crosslinking between the interpolar and kinetochore fibers (Matthies et al. 1996, Jang et al. 2005). The NCD motor protein may be an agent in this crosslinking, possibly in the following fashion: the motor may be in contact with both the interpolar and kinetochore fibers as it moves between them, thereby guiding the kinetochore fibers in the two poleward directions established by the central spindle. A corollary of this model is that the interpolar microtubules of the central spindle must be present before the kinetochore microtubules can be organized. 
In summary, the recruitment of the microtubules by the chromosomes, possibly involving lateral interactions, the establishment of the polar axis by the precociously formed central spindle, and the action of minus-end motors like NCD, appear to function together to form the acentrosomal meiotic spindle. They act in an 'inside-out' process, driving spindle formation from the middle that is, from the chromosomes, toward the poles. This process appears to compensate for the absence of centrosomes and predefined spindle poles. It is also a model which could assemble a bipolar spindle without kinetochore microtubules. Surprisingly, even though a bipolar spindle forms, there is considerable movement of the chromosomes, suggesting a metaphase spindle forms prior to stable bipolar attachment of the chromosomes (Skold et al. 2005). Although it is not known how the kinetochores obtain bipolar attachments, there is evidence the homologs orient prior to assembly of the bipolar spindle (Jang et al. 2005).

The spindle poles: MSPS, TACC and NCD (and possibly ASP) are required for maintaining the oocyte spindle poles

Even though the metaphase I spindle poles of Drosophila oocytes lack centrosomal antigens, they are not devoid of structure. Several proteins localize to the meiotic acentrosomal poles, including transforming acidic coiled-coil protein (TACC), minispindles (MSPS) (Cullen \& Ohkura 2001) and abnormal spindle (ASP) (Riparbelli et al. 2002). Although important for focusing spindle poles in mitotic cells (Wakefield et al. 2001), the role of Asp in acentrosomal meiosis, as currently understood, is to assist during early telophase I to reorganize the microtubules for the assembly of the two tandem spindles of meiosis II (Riparbelli et al. 2002).

MSPS is a member of the dis1/TOG family, a group of conserved microtubule-associated proteins. In mitosis, MSPS is found to localize to centrosomes where it promotes microtubule growth and stabilizes microtubule ends, thereby playing an important role in mitotic spindle integrity (Lee et al. 2001). But in the absence of centrosomes in oocyte meiosis, MSPS is still able to localize to the spindle poles. Loss of MSPS disrupts the bipolarity of acentrosomal meiotic spindles, resulting in a predominance of tripolar morphology (Cullen \& Ohkura 2001).
MSPS is not likely to function in the initial assembly of the bipolar spindle, as it is seen at the poles only after the bipolar spindle is assembled. The tripolar spindle phenotype of the msps mutant suggests that it may act to maintain bipolarity. Because dis1/TOG family members are known to promote microtubule growth and stabilize ends (McNally 2003), and because MSPS is found at the acentrosomal poles, Cullen and Ohkura proposed that microtubule fibers connected to the established poles are preferentially stabilized, while the re-growth of microtubules that are detached from the two established poles is not promoted (Cullen \& Ohkura 2001). In this way the primary function of Msps may be to maintain spindle poles and bipolarity.

Given the absence of centrosomes, how do proteins like TACC and MSPS get to the poles? Polar localization of MSPS in stage 14 oocytes is mediated by the NCD kinesin-like protein and TACC (Cullen \& Ohkura 2001). TACC has been found to localize to the poles even before metaphase arrest, while MSPS is seen at the poles slightly later, after bipolarity has been clearly established. TACC is essential for maintaining MSPS at the poles. The observation that the NCD motor is needed for efficient localization of MSPS to the poles (Cullen \& Ohkura 2001) suggests that some proteins are moved to the poles. How TACC becomes concentrated to the meiotic acentrosomal poles is not known.

These results demonstrate that, while the organization of microtubules may not be initiated at the acentrosomal poles, these poles still have proteins associated with them and still perform functions important for stabilizing minus ends and maintaining the two poles. Moreover, there is a synergistic effect on spindle formation in sub; tacc double mutants (Jang et al. 2005). This suggests that there are two separate pathways in acentrosomal spindle formation. Subito is associated with the central spindle and the establishment of bipolarity, while TACC is associated with maintenance of the poles.

\section{Specialized tubulin isoforms}

Mutations in some tubulin genes result in female sterility but the flies are otherwise viable. These appear to be tubulin isoforms with female-specific functions. One of these is a $\gamma$-tubulin isoform $(\gamma$-TUB37C $)$ that, while not present at the acentrosomal spindle 
poles of the oocyte, appears to be required for bipolar spindle assembly. Oocytes deficient or totally lacking in $\gamma$-TUB37C exhibit disorganized spindles at stage 14 (Tavosanis et al. 1997, Jang et al. 2005). Similarly, there is a female-specific $\alpha$-tubulin which is also required for acentrosomal spindle assembly and fertility (Matthews et al. 1993, Jang et al. 2005). Perhaps these female-specific tubulin isoforms have roles specific to acentrosomal spindle assembly.

\section{The achiasmate segregation system}

Drosophila females possess a mechanism to accurately segregate achiasmate homologs (Hawley \& Theurkauf 1993). This system is intimately associated with the meiotic spindle, and many of the mutants described above are defective for the segregation of achiasmate homologs. Indeed, it seems the segregation of achiasmate chromosomes is more sensitive than chiasmate chromosomes to spindle defects. Some mutants have been identified in which their effects on the achiasmate segregation are more severe than on chiasmate chromosome segregation. These studies have led to a model which includes achiasmate systemspecific concepts of pairing, orientation, and disjunction (Carpenter 1973, Hawley \& Theurkauf 1993).

Pairing of achiasmate chromosomes appears to be mediated by heterochromatic homology (Hawley et al. 1992, Dernburg et al. 1996). The heterochromatic pairings can persist until prometaphase but are dissolved earlier in prophase when the homologous chromosomes undergo crossing over (Xiang \& Hawley 2006). Thus, crossovers influence heterochromatic pairing and, as a result, determine whether a chromosome will orient as part of the chiasmate or achiasmate systems.

An example of a gene required for pairing or orientation is Matrimony, which was isolated as a haploinsufficient locus. It is required for achiasmate chromosomes to be properly positioned with respect to their homolog on the meiotic spindle, possibly acting as some sort of glue (Harris et al. 2003). Also involved in pairing or orientation is ald (altered disjunction), mutants of which cause nondisjunction of both achiasmate and chiasmate chromosomes. Interestingly, ALD is an ortholog of Mps1 in mammals, a protein associated with checkpoint function (Gilliland et al. 2005). Thus, this is the first evidence that a spindle assembly checkpoint mechanism has a role in the segregation of chromosomes on acentrosomal spindles in Drosophila.

NOD (no distributive disjunction) is a chromokinesin-like protein (a chromosome-associated kinesinlike protein) that localizes along the arm of meiotic chromosomes (Afshar et al. 1995a,b, Cui \& Hawley 2005) and is only required for achiasmate disjunction. Without NOD, achiasmate chromosomes have been shown to dissociate from the karyosome immediately after NEB (Theurkauf \& Hawley 1992, McKim \& Hawley 1995). By binding to microtubule plus-ends and promoting their growth, NOD may be able to hold chromosomes near the metaphase plate, opposing the poleward force exerted on the kinetochores (Cui et al. 2005).

Another gene that may be in the disjunction class is aberrant $\mathrm{X}$ segregation (AXS), which encodes a transmembrane protein that is recruited to the microtubules of assembling spindles in both mitotic and meiotic cells (Kramer \& Hawley 2003). After NEB, AXS localizes to the outer surface of the karyosome, and remains associated with the developing spindle through all stages of assembly, principally at the central spindle area. It forms part of a membranous structure that provides a sheath for the spindle. Part of the oocyte maturation process, AXS is proposed to be a mediator of meiotic spindle assembly and chromosome segregation.

\section{Mus musculus}

\section{Meiosis overview in the mouse oocyte}

In the oocytes of mammals, synapsis and meiotic recombination occur during maternal fetal development, after which the oocyte enters prophase I arrest, or the dictyate stage (Hassold \& Hunt 2001, Mehlmann 2005). Following this, the immature oocyte, encased as part of the primordial follicle within a somatic cell, enters a growth stage. Once the female is sexually mature, in humans years after it began meiosis, the oocyte completes meiosis I just before ovulation.

At this point germinal vessel breakdown (GVBD), the mammalian equivalent of Drosophila's NEB, takes place and the bipolar spindle forms. Assembly of the spindle microtubules is regulated by the chromosomes, although during most of prometaphase, lateral interactions between chromosome-based 
chromokinesin molecules and microtubules may be more important than kinetochore interactions with the microtubules. In mice the assembly of the bipolar spindle takes about $4 \mathrm{~h}$, depending on genetic background (Brunet et al. 1999). The spindle is then maintained in prometaphase I for another $4 \mathrm{~h}$, with the chromosomes hovering in the spindle's central region. Only at the end of this period, $8 \mathrm{~h}$ after GVBD, do the kinetochores become activated so that microtubule fibers can stably connect to them. Then the chromosomes align at the metaphase plate and anaphase I ensues. Steady alignment of the chromosomes at the metaphase plate occurs for only about $12 \mathrm{~min}$ at metaphase I.

Microtubules polymerize into bipolar spindles, but chromosomes control their number and size

Szollosi et al. (1972) used electron microscopy to document the lack of centrioles at the resumption of meiosis I in mouse oocytes. They observed small 'asterlike' radial structures, referred to as 'microtubule foci', and characterized them as 'electron-dense, filamentous structures resembling diffuse centriolar satellites'. Maro et al. (1985), studying metaphase IIarrested mouse oocytes, found similar microtubule foci forming near chromosomes, which they described as consisting of pericentriolar material. An interesting possibility is that these structures represent small microtubule 'factories' dispersed throughout the cytoplasm (Figure 1). Similar structures may exist in Drosophila oocytes (Skold et al. 2005).

Mouse oocytes appear to be capable of polymerizing microtubules and organizing them into multiple bipolar structures not only without centrosomes but also in the absence of chromosomes. This has been demonstrated in studies using cytoplasts; that is, oocyte cytoplasm lacking the nucleus (Brunet et al. 1998). Multipolar spindles were found in addition to bipolar spindles, but in lower numbers, suggesting that bipolar spindles may be the most stable. However, while chromosomes may not be essential for the polymerization of microtubules, they appear to regulate the numbers and sizes of the spindles. In the mouse cytoplasts several bipolar spindles tended to form, indicating that chromosomes may be needed to restrict spindle formation (Brunet et al. 1999). Furthermore, in cytoplasts the size of the spindles varied, suggesting that chromosomes control spindle growth, possibly through the activation of microtubule-stabilizing factors.

The balanced arrangement of homologous chromosomes within the spindle has been demonstrated to stabilize the formation of the spindle poles. In mice deficient in the DNA mismatch repair protein MLH1 (mutL homolog 1), crossing over in oocytes is sharply reduced (Baker et al. 1996), resulting at prometaphase I in univalent chromosomes that fail to establish bipolar spindle attachments. In these oocytes the meiosis I spindles are unusually long and anaphase I generally fails to take place (Woods et al. 1999). Therefore, the attachment of chiasmate homologous chromosomes to opposite poles may help stabilize the bipolar spindle.

As discussed above for Drosophila (McKim et al. 1993), tension created when bivalents achieve bipolar attachment could contribute to spindle stability. Kinetochores under tension may act to stabilize the metaphase I spindle rather than promote anaphase, as occurs in mitotic cells. Woods et al. (1999) made the intriguing suggestion that bipolar attachments are being made as the spindle develops, based on an abnormal rosette configuration of univalent chromosomes observed at the periphery of a microtubule mass in early prometaphase. In $m l h$ mutants, approximately equal numbers of the univalent chromosomes oriented toward each pole, though this balance is a matter of numbers, not homologies. As is the case of Drosophila, the meiotic chromosomes in mammals may position themselves prior to the organization of the bipolar spindle, possibly by interacting with non-kinetochore microtubules.

\section{RanGTP and the effect of chromosomes on spindle assembly}

The contribution of chromosomes to the assembly of the spindle, both with and without centrosomes, is believed to be linked to the activity of the GTPase Ran (Karsenti \& Vernos 2001). In the presence of a chromatin-associated protein RCC1, RanGDP is converted into its active form RanGTP. Due to the restriction of RCC1 localization, active RanGTP is concentrated near the chromosomes where it locally activates spindle assembly promoting factors such as TPX2 and XMAP215 (Gruss \& Vernos 2004). Interestingly, Ran has been observed around mouse oocyte chromosomes and has been conjectured to play a role in meiotic spindle assembly (Brunet \& 
Maro 2005, Cao et al. 2005). These properties suggest Ran signaling may mediate many of the processes by which meiotic chromosomes influence acentrosomal spindle assembly. In Xenopus extracts a gradient of RanGTP has been shown to promote microtubule nucleation and spindle assembly (e.g. Ohba et al. 1999, Brunet et al. 2004, Caudron et al. 2005). In mouse oocytes, however, the data is conflicting. In one study based on antibody microinjection, defects in meiosis I spindle assembly were observed (Cao et al. 2005). In contrast, Dumont et al. (2007), in a study based on manipulating RanGTP levels by expressing mutant forms of Ran, showed that the most severe spindle assembly defects were observed during meiosis II. There were only minor effects on meiosis I spindles, such as on spindle length and timing, but they were still bipolar and there was no effect on chromosome segregation at the reductional division.

\section{Kinetochore-microtubule interactions do not occur in oocytes during Prometaphase I}

In mitosis the interaction of kinetochores and microtubules is a key factor in the congression of chromosomes toward the metaphase plate during prometaphase. In mouse oocytes, however, Brunet et al. (1999) observed that kinetochores do not interact directly with microtubules until the end of prometaphase I, about $8 \mathrm{~h}$ after GVBD. Thus, the kinetochores are not competent to anchor and stabilize the microtubules until late in prometaphase. The lack of kinetochore-microtubule interaction during the first several hours of prometaphase was observed in two ways: directly using electron microscopy and by following the localization of the protein CLIP-170, which disassociates from kinetochores in the presence of microtubules. Since Brunet et al. found CLIP-170 at the kinetochores up until $7 \mathrm{~h}$ after GVBD, they concluded that the kinetochore microtubules were absent during this period. Indeed, for the approximately $4 \mathrm{~h}$ duration of prometaphase I, the microtubules come into lateral contact with the surface of the chromosomes and in some instances penetrate the chromatin, without directly interacting with the kinetochores.

With a duration of about $4 \mathrm{~h}$, late prometaphase I in mouse oocytes is relatively long; and despite the lack of kinetochore-microtubule interaction to stabilize the chromosomes in the vicinity of the meta- phase plate, the chromosomes do oscillate very near the spindle equator during this period (Brunet et al. 1999, Woods et al. 1999). It was speculated that the chromosomes are held near the equator through the exertion of polar ejection forces acting on the chromosome arms by spindle microtubules, mediated by chromatin-associated microtubule motors (Khodjakov \& Rieder 1996, Brunet \& Vernos 2001). These motors include Kif4 and Kif22 (orthologs of Xenopus kinesinlike proteins Xklp1 and Xkid) (Vernos et al. 1995) which may facilitate bivalent congression in oocyte meiosis. In Drosophila, as discussed above, the chromokinesin protein NOD has been shown to exert an anti-poleward force upon the chromosomes.

\section{$M P F$ activity and synchronizing events leading to spindle formation and the accurate alignment of bivalents}

MPF, the maturation-promoting factor, regulates the sequence and timing of meiotic events, notably maturation, the formation of spindles, asymmetry of the divisions and polar body extrusion. MPF levels begin to increase at GVBD, reach a plateau at the end of metaphase I, decline between metaphase I and II for polar body extrusion and return to high levels during metaphase II arrest (Brunet \& Maro 2005). It is tempting to speculate that the increasing levels of MPF may trigger two phases of spindle assembly. Once MPF increases to a first threshold the microtubules are able to organize into a bipolar structure. By the end of prometaphase I MPF levels have increased to a second threshold, which correlates with activation of the kinetochores (Polanski et al. 1998, Brunet et al. 1999). This enables the capture of the microtubules by the kinetochores, and the assembly of the kinetochore fibers. Once this occurs, accurate metaphase plate alignment can take place.

The spindle assembly checkpoint exists in female meiosis but its effectiveness is in question

Meiosis in human oocytes is notoriously error-prone, and the incidence of these errors increases with maternal age. This has been well documented from looking at the origin of human aneuploidy, especially trisomies (Hassold \& Hunt 2001). Although the incidence varies from chromosome to chromosome, aneuploidy usually results from nondisjunction at meiosis I in the oocyte. A minimum estimate for the 
frequency of aneuploidy in oocytes is $20 \%$, compared to between $1 \%$ and $2 \%$ in sperm (Hassold \& Hunt 2001). Several explanations have been proposed for this high rate of nondisjunction in oocytes, including the long time between prophase and metaphase, the lack of centrosomes, and an inefficient spindle assembly checkpoint (SAC).

But several studies indicate the SAC does function in mammalian oocytes. Known checkpoint proteins BUB1 and MAD2 are present on kinetochores during oocyte meiosis. BUB1 is associated with kinetochores from prometaphase I until early anaphase I and again during the metaphase II arrest in mouse oocytes, suggesting that it may have a role in the natural progression of the two meiotic divisions (Brunet et al. 2003). Consistent with this, BUB1 is in a phosphorylated state throughout this time, typical of when the SAC activated. In contrast, MAD2 is present early in prometaphase (Wassmann et al. 2003). MAD2 may also have important functions in the absence of spindle damage, as progression through meiosis I was accelerated (Tsurumi et al. 2004) and there was an increase in chromosome segregation errors when MAD2 was depleted (Homer et al. 2005b).

When microtubule dynamics are altered using nocodazole or taxol, several events occur which suggest the SAC is activated. MAD2, which normally disappears from the kinetochores during prometaphase, relocalizes upon spindle damage induced by nocodazole (Wassmann et al. 2003). Meiotic progression is also affected, as the drop of MPF between meiosis I and II is inhibited and anaphase I onset is delayed (Brunet et al. 2003). As expected for a checkpoint function, this arrest was reversible when the drug was washed away from the oocytes (Wassmann et al. 2003). Other studies have indicated that the oocyte spindle assembly checkpoint in meiosis I depends on the action of MAD2. Depletion of MAD2 from oocytes leads to a failure to respond to spindle damage (Homer et al. 2005a, Wassmann et al. 2003).

Despite this evidence for the existence of the SAC, the high rate of nondisjunction in mammalian female meiosis calls into question the effectiveness of the $\mathrm{SAC}$ to detect chromosomal misalignment within the spindle. If indeed oocyte meiosis includes a SAC that is intended to delay anaphase onset when kinetochore attachment is faulty, then this mechanism may be weak or subject to frequent breakdown. For example, the effectiveness of the SAC could be limited since mouse oocyte arrest is a fleeting event (Wassmann et al. 2003), at least at low doses of nocodozole (Homer et al. 2005a). Alternatively, the oocyte SAC may not detect chromosome alignment errors. As suggested by Woods et al. (1999), the oocyte SAC may only detect structural abnormalities in the spindle, as would be caused by the various drugs which destabilize or stabilize microtubules. In contrast, unattached chromosomes or univalents attached to only one pole on the spindle appear not to trigger an arrest (LeMaire-Adkins et al. 1997, LeMaire-Adkins \& Hunt 2000).

\section{Summary and the future}

Chromosome mis-segregation in meiosis I of oocytes is believed to be the major cause of miscarriage and congenital birth defects. This strongly indicates that female meiosis, in which acentrosomal spindle assembly is a major distinguishing factor, is worthy of increased examination. The studies summarized here demonstrate that organisms as diverse as Drosophila and the mouse may have dealt with this problem utilizing similar solutions. A hurdle toward more direct comparisons of the systems, however, is that most of the genes studied in one system have not been studied in the other.

\section{Initial assembly of the meiosis I spindle}

At the onset of meiosis I, just after nuclear envelope breakdown (NEB) in Drosophila oocytes and germinal vesicle breakdown (GVBD) in mammalian oocytes, microtubules begin to organize in the absence of centrosomes. In both organisms the chromosomes appear to be instrumental in initiating and regulating spindle assembly. Paradoxically, the important microtubule contacts may not be with kinetochores. Instead, microtubule contacts with the chromosome arms, perhaps laterally, may be important. The results from mouse cytoplasts show that microtubules are able to polymerize and organize into spindles without chromosomes, and there are similar observations in Drosophila oocytes (J. K. Jang $\&$ K. S. McKim unpublished). Thus, the chromosomes may play a restraining role in pole formation. Microtubules could be preferentially assembled into bundles in the region near the chromosomes. 
Eventual attachment of the chromosomes to opposite poles via kinetochore microtubules, however, is instrumental in stabilizing the bipolar spindle.

\section{Where do the microtubules come from?}

There is no evidence that the chromosomes nucleate microtubule fiber growth. Instead, it is likely that microtubules may pre-exist in the cytoplasm (Figure 2). They may grow from small organizing centers, as has been suggested by the observations of aster-like structures in mouse (Szollosi et al. 1972) and Drosophila oocytes (Skold et al. 2005). These nucleating centers could be numerous. Microtubule growth is nucleated from many organizing centers along the cortex of Drosophila oocytes prior to nuclear envelop breakdown as part of the axis specification during oogenesis (Theurkauf et al. 1992, Cha et al. 2002, Steinhauer \& Kalderon 2006). Assembling the spindle, therefore, could be primarily done by the bundling and sliding of microtubule fibers, the result of which would be a structure composed of an overlapping array of many short fibers. This predicts that microtubule plus and minus ends would be localized throughout meiotic spindles, and indeed there is evidence this is the case (Elliott et al. 2005, Burbank et al. 2006).

Surprisingly, the severing of microtubules may be an important regulator of spindle assembly in $C$. elegans oocytes. Katanin is a conserved ATPase known to have a microtubule-severing function (McNally \& Vale 1993). Srayko et al. (2006) suggest that katanin may be needed in acentrosomal meiosis to compensate for the relative inefficiency of chromatin-based microtubule nucleation. Microtubule fragments may act as seeds for microtubule growth (Ribbeck \& Mitchison 2006).

\section{On the relative roles of two types of fibers}

The interpolar microtubules organize tangentially to the karyosome, and by metaphase I run from pole to pole. The meiotic central spindle has been demonstrated in Drosophila to form early in prometaphase I, earlier than in mitosis, possibly to establish the axis of chromosomal separation, and thus the relative locations of the poles. The central spindle may 'communicate' with the kinetochore microtubules using crosslinking motors such as NCD. Bundling of microtubules is also important in organizing the
$C$. elegans spindle. In $C$. elegans oocytes the depletion of KLP-18, which is similar to Xenopus kinesin Klp2, results in the inhibition of parallel bundling of microtubules around the chromosomes, and disturbs the organization of the bipolar spindle (Segbert et al. 2003).

Whether interpolar microtubules and the central spindle are important organizers in mouse oocytes and the roles for orthologs of SUB and NCD in bundling microtubules remains to be determined. Interpolar microtubules are more sensitive to temperature and microtubule-depolymerizing drugs than kinetochore microtubules (Brinkley \& Cartwright 1975, Salmon \& Begg 1980, J. K. Jang \& K. S. McKim unpublished). Thus, investigating these microtubules in mouse oocytes may provide insights into spindle assembly and highlight a possible weakness that could lead to segregation errors.

In mouse oocytes the microtubules attach to the kinetochores relatively late in prometaphase. It will be interesting to determine if this is also true in Drosophila, especially since in both systems the kinetochores are not needed for spindle formation. There is evidence suggesting that the mouse oocyte chromosomes are transported to the spindle equator not by spindles attached to the kinetochores, as in mitosis, but by the action of pushing forces by chromokinesin motors along microtubules. In Drosophila the chromokinesin NOD acts in a similar fashion.

\section{How do the bivalents orient?}

In both mouse and Drosophila oocytes there is evidence that the homologs orient prior to bipolar spindle formation. This may be necessary if, as seems likely, spindle assembly does not initially involve kinetochore microtubules. In addition, the chromosomes oscillate near the spindle equator after a bipolar spindle has formed. It thus seems likely that bivalents on acentrosomal spindles orient relative to the poles by a mechanism which differs from centrosomal spindles as described in the Introduction.

\section{The meiotic spindle poles}

Centrosomes may be lacking, but the meiotic spindle poles can have specific proteins associated with them. MSPS appears to promote spindle bipolarity; and NCD and TACC seem to promote, respectively, 
the localization and the maintenance of MSPS at the poles. The mouse oocyte spindle poles, like those in many acentrosomal systems, are broad. In contrast, Drosophila meiotic spindle poles are unusually well defined. It remains to be determined if the mouse oocyte spindle poles contain proteins found at mitotic spindle poles, just like Drosophila, or whether this is a potential weak point.

\section{Meiotic checkpoints in the oocyte}

The mitotic spindle assembly checkpoint (SAC) delays anaphase until all the homologous kinetochores have bipolar attachments on the spindle. In human female meiosis a high incidence of nondisjunction suggests that this checkpoint is not as rigorous in oocytes as in sperm. However, evidence in mouse oocytes indicates that the SAC does indeed exist in female meiosis. In contrast, virtually nothing is known about the SAC in Drosophila oocytes, although the first mutants in genes such as mad2 and mpsl are now being studied. Most important, however, in both Drosophila and mouse, the presence of unattached chromosomes, such as univalents, does not appear to delay the onset of anaphase. This disconnect between spindle assembly and chromosome orientation and attachment could be a major contributor to the high error rate in oocytes.

\section{Model systems and nondisjunction in humans}

There are two critical characteristics of meiosis in human females that are particularly important: the high frequency of nondisjunction and its further increase with advancing age. Unfortunately, the model systems reviewed here do not usually show these features with such magnitude. Nonetheless, the model organism studies may identify possible 'weak links' in human female meiosis. This includes the features of acentrosomal spindle assembly such as a heavy reliance on interpolar microtubules, a weaker SAC and broad spindle poles. There are also other factors which may play a tangential role to spindle assembly. Sister chromatid cohesion, which is also required during meiosis I to maintain chiasma, has been suggested to be a link to age-related nondisjunction (Hodges et al. 2005, Bickel 2005). Nondisjunction dramatically increases in mouse interspecific hybrids, indicating that sequence variation can contribute to the frequency of nondisjunction
(Koehler et al. 2006), although this may not be the case in Drosophila (Rutherford \& Carpenter 1988). Finally, it is perhaps the sum of several factors that lead to high frequency of nondisjunction in human females.

\section{Acknowledgements}

We are grateful to Janet Jang for Figure 2 and Sarah Radford and Jeffry Cesario for critical reading of the manuscript. This work was supported by a grant from the National Institutes of Health (GM 067142) to K. McKim.

\section{References}

Afshar K, Barton NR, Hawley RS, Goldstein LSB (1995a) DNA binding and meiotic chromosomal localization of the Drosophila Nod kinesin-like protein. Cell 81: 129-138.

Afshar K, Scholey J, Hawley RS (1995b) Identification of the chromosome localization domain of the Drosophila Nod kinesin-like protein. J Cell Biol 131: 833-843.

Baker SM, Plug AW, Prolla TA et al. (1996) Involvement of mouse Mlh1 in DNA mismatch repair and meiotic crossing over. Nat Genet 13: 336-342.

Bickel SE (2005) Aging (not so) gracefully. Nat Genet 37: 1303-1304.

Bloch Qazi MC, Heifetz Y, Wolfner MF (2003) The developments between gametogenesis and fertilization: ovulation and female sperm storage in Drosophila melanogaster. Dev Biol 256: 195-211.

Brent AE, MacQueen A, Hazelrigg T (2000) The Drosophila wispy gene is required for RNA localization and other microtubulebased events of meiosis and early embryogenesis. Genetics 154 : 1649-1662.

Brinkley BR, Cartwright J Jr (1975) Cold-labile and cold-stable microtubules in the mitotic spindle of mammalian cells. Ann NY Acad Sci 253: 428-439.

Brunet S, Maro B (2005) Cytoskeleton and cell cycle control during meiotic maturation of the mouse oocyte: integrating time and space. Reproduction 130: 801-811.

Brunet S, Vernos I (2001) Chromosome motors on the move. From motion to spindle checkpoint activity. EMBO Rep 2: 669-673.

Brunet S, Maria AS, Guillaud P, Dujardin D, Kubiak JZ, Maro B (1999) Kinetochore fibers are not involved in the formation of the first meiotic spindle in mouse oocytes, but control the exit from the first meiotic M phase. J Cell Biol 146: 1-12.

Brunet S, Pahlavan G, Taylor S, Maro B (2003) Functionality of the spindle checkpoint during the first meiotic division of mammalian oocytes. Reproduction 126: 443-450.

Brunet S, Polanski Z, Verlhac MH, Kubiak JZ, Maro B (1998) Bipolar meiotic spindle formation without chromatin. Curr Biol 8: 1231-1234. 
Brunet S, Sardon T, Zimmerman T et al. (2004) Characterization of the TPX2 domains involved in microtubule nucleation and spindle assembly in Xenopus egg extracts. Mol Biol Cell 15: 5318-5328.

Burbank KS, Groen AC, Perlman ZE, Fisher DS, Mitchison TJ (2006) A new method reveals microtubule minus ends throughout the meiotic spindle. J Cell Biol 175: 369-375.

Cao YK, Zhong ZS, Chen DY, Zhang GX, Schatten H, Sun QY (2005) Cell cycle-dependent localization and possible roles of the small GTPase Ran in mouse oocyte maturation, fertilization and early cleavage. Reproduction 130: 431-440.

Carpenter ATC (1973) A meiotic mutant defective in distributive disjunction in Drosophila melanogaster. Genetics 73: 393-428.

Caudron M, Bunt G, Bastiaens P, Karsenti E (2005) Spatial coordination of spindle assembly by chromosome-mediated signaling gradients. Science 309: 1373-1376.

Cha BJ, Serbus LR, Koppetsch BS, Theurkauf WE (2002) Kinesin I-dependent cortical exclusion restricts pole plasm to the oocyte posterior. Nat Cell Biol 4: 592-598.

Chandra R, Salmon ED, Erickson HP, Lockhart A, Endow SA (1993) Structural and functional domains of the Drosophila ncd microtubule motor protein. J Biol Chem 268: 9005-9013.

Compton DA (1998) Focusing on spindle poles. J Cell Sci 111: 1477-1481.

Compton DA (2000) Spindle assembly in animal cells. Annu Rev Biochem 69: 95-114.

Cui W, Hawley RS (2005) The HhH2/NDD domain of the Drosophila Nod chromokinesin-like protein is required for binding to chromosomes in the oocyte nucleus. Genetics 171: 1823-1835.

Cui W, Sproul LR, Gustafson SM, Matthies HJ, Gilbert SP, Hawley RS (2005) Drosophila Nod protein binds preferentially to the plus ends of microtubules and promotes microtubule polymerization in vitro. Mol Biol Cell 16: 5400-5409.

Cullen CF, Ohkura H (2001) Msps protein is localized to acentrosomal poles to ensure bipolarity of Drosophila meiotic spindles. Nature Cell Biol 3: 637-642.

Cullen CF, Brittle AL, Ito T, Ohkura H (2005) The conserved kinase NHK-1 is essential for mitotic progression and unifying acentrosomal meiotic spindles in Drosophila melanogaster. J Cell Biol 171: 593-602.

D’Avino PP, Savoian MS, Glover DM (2005) Cleavage furrow formation and ingression during animal cytokinesis: a microtubule legacy. J Cell Sci 118: 1549-1558.

Dernburg AF, Sedat JW, Hawley RS (1996) Direct evidence of a role for heterochromatin in meiotic chromosome segregation. Cell 85: 135-146.

Dumont J, Petri S, Pellegrin F et al. (2007) A centriole- and RanGTP-independent spindle assembly pathway in meiosis I of vertebrate oocytes. J Cell Biol 176: 295-305.

Elliott SL, Cullen CF, Wrobel N, Kernan MJ, Ohkura H (2005) EB1 is essential during Drosophila development and plays a crucial role in the integrity of chordotonal mechanosensory organs. Mol Biol Cell 16: 891-901.

Endow SA, Komma DJ (1997) Spindle dynamics during meiosis in Drosophila oocytes. J Cell Biol 137: 1321-1336.

Giansanti MG, Bonaccorsi S, Williams B et al. (1998) Cooperative interactions between the central spindle and the contractile ring during Drosophila cytokinesis. Genes Dev 12: 396-410.
Gilliland WD, Wayson SM, Hawley RS (2005) The meiotic defects of mutants in the Drosophila mps1 gene reveal a critical role of Mps1 in the segregation of achiasmate homologs. Curr Biol 15: 672-677.

Giunta KL, Jang JK, Manheim EM, Subramanian G, McKim KS (2002) subito encodes a kinesin-like protein required for meiotic spindle pole formation in Drosophila melanogaster. Genetics 160: 1489-1501.

Goshima G, Nedelec F, Vale RD (2005) Mechanisms for focusing mitotic spindle poles by minus end-directed motor proteins. J Cell Biol 171: 229-240.

Gruss OJ, Vernos I (2004) The mechanism of spindle assembly: functions of Ran and its target TPX2. J Cell Biol 166: 949-955.

Hall H, Hunt P, Hassold T (2006) Meiosis and sex chromosome aneuploidy: how meiotic errors cause aneuploidy; how aneuploidy causes meiotic errors. Curr Opin Genet Dev 16: 323-329.

Harris D, Orme C, Kramer J et al. (2003) A deficiency screen of the major autosomes identifies a gene (matrimony) that is haploinsufficient for achiasmate segregation in Drosophila oocytes. Genetics 165: 637-652.

Hassold T, Hunt P (2001) To err (meiotically) is human: the genesis of human aneuploidy. Nat Rev Genet 2: 280-291.

Hatsumi M, Endow SA (1992) The Drosophila ncd microtubule motor protein is spindle- associated in meiotic and mitotic cells. J Cell Sci 103: 1013-1020.

Hawley RS, Theurkauf WE (1993) Requiem for distributive segregation: achiasmate segregation in Drosophila females. Trends Genet 9: 310-317.

Hawley RS, Irick HA, Zitron AE et al. (1992) There are two mechanisms of achiasmate segregation in Drosophila females, one of which requires heterochromatic homology. Dev Genet 13: $440-467$.

Hodges CA, Revenkova E, Jessberger R, Hassold TJ, Hunt PA (2005) SMC1beta-deficient female mice provide evidence that cohesins are a missing link in age-related nondisjunction. Nat Genet 37: 1351-1355.

Homer HA, McDougall A, Levasseur M, Murdoch AP, Herbert M (2005a) Mad2 is required for inhibiting securin and cyclin B degradation following spindle depolymerisation in meiosis I mouse oocytes. Reproduction 130: 829-843.

Homer HA, McDougall A, Levasseur M, Yallop K, Murdoch AP, Herbert M (2005b) Mad2 prevents aneuploidy and premature proteolysis of cyclin B and securin during meiosis I in mouse oocytes. Genes Dev 19: 202-207.

Ivanovska I, Lee E, Kwan KM, Fenger DD, Orr-Weaver TL (2004) The Drosophila MOS ortholog is not essential for meiosis. Curr Biol 14: 75-80.

Jang JK, Messina L, Erdman MB, Arbel T, Hawley RS (1995) Induction of metaphase arrest in Drosophila oocytes by chiasma-based kinetochore tension. Science 268: 1917-1919.

Jang JK, Rahman T, McKim KS (2005) The kinesin-like protein Subito contributes to central spindle assembly and organization of the meiotic spindle in Drosophila oocytes. Mol Biol Cell 16: 4684-4694.

Jeffreys CA, Burrage PS, Bickel SE (2003) A model system for increased meiotic nondisjunction in older oocytes. Curr Biol 13: 498-503.

Karsenti E, Newport J, Hubble R, Kirschner M (1984a) Interconversion of metaphase and interphase microtubule arrays, as 
studied by the injection of centrosomes and nuclei into Xenopus eggs. J Cell Biol 98: 1730-1745.

Karsenti E, Newport J, Kirschner M (1984b) Respective roles of centrosomes and chromatin in the conversion of microtubule arrays from interphase to metaphase. J Cell Biol 99: 47s-54s.

Karsenti E, Vernos I (2001) The mitotic spindle: a self-made machine. Science 294: 543-547.

Khodjakov A, Rieder CL (1996) Kinetochores moving away from their associated pole do not exert a significant pushing force on the chromosome. J Cell Biol 135: 315-327.

King RC (1970) Ovarian Development in Drosophila melanogaster. New York: Academic Press.

Kirschner M, Mitchison T (1986) Beyond self-assembly: from microtubules to morphogenesis. Cell 45: 329-342.

Koehler KE, Schrump SE, Cherry JP, Hassold TJ, Hunt PA (2006) Near-human aneuploidy levels in female mice with homeologous chromosomes. Curr Biol 16: R579-580.

Kramer J, Hawley RS (2003) The spindle-associated transmembrane protein Axs identifies a membranous structure ensheathing the meiotic spindle. Nat Cell Biol 5: 261-263.

Lee MJ, Gergely F, Jeffers K, Peak-Chew SY, Raff JW (2001) Msps/XMAP215 interacts with the centrosomal protein D-TACC to regulate microtubule behavior. Nat Cell Biol 3: 643-649.

Lemaire-Adkins R, Hunt PA (2000) Nonrandom segregation of the mouse univalent $\mathrm{X}$ chromosome: evidence of spindle-mediated meiotic drive. Genetics 156: 775-783.

Lemaire-Adkins R, Radke K, Hunt PA (1997) Lack of checkpoint control at the metaphase/anaphase transition: a mechanism of meiotic nondisjunction in mammalian females. J Cell Biol 139: 1611-1619.

Maro B, Howlett SK, Webb M (1985) Non-spindle microtubule organizing centers in metaphase II-arrested mouse oocytes. $J$ Cell Biol 101: 1665-1672.

Matthews KA, Rees D, Kaufman TC (1993) A functionally specialized a-tubulin is required for oocyte meiosis and cleavage mitoses in Drosophila. Development 117: 977-991.

Matthies HJ, McDonald HB, Goldstein LS, Theurkauf WE (1996) Anastral meiotic spindle morphogenesis: role of the non-claret disjunctional kinesin-like protein. J Cell Biol 134: 455-464.

McDonald HB, Stewart RJ, Goldstein LSB (1990) The kinesin-like ncd protein of Drosophila is a minus end-directed microtubule motor. Cell 63: 1159-1165.

McKim KS, Hawley RS (1995) Chromosomal control of meiotic cell division. Science 270: 1595-1601.

McKim KS, Jang JK, Theurkauf WE, Hawley RS (1993) Mechanical basis of meiotic metaphase arrest. Nature 362: 364-366.

McNally F (2003) Microtubule dynamics: new surprises from an old MAP. Curr Biol 13: R597-599.

McNally FJ, Vale RD (1993) Identification of katanin, an ATPase that severs and disassembles stable microtubules. Cell 75: 419-429.

McNally KL, McNally FJ (2005) Fertilization initiates the transition from anaphase I to metaphase II during female meiosis in C. elegans. Dev Biol 282: 218-230.

Mehlmann LM (2005) Stops and starts in mammalian oocytes: recent advances in understanding the regulation of meiotic arrest and oocyte maturation. Reproduction 130: 791-799.
Morelli MA, Cohen PE (2005) Not all germ cells are created equal: aspects of sexual dimorphism in mammalian meiosis. Reproduction 130: 761-781.

Nicklas RB (1997) How cells get the right chromosomes. Science 275: 632-637.

Nislow C, Lombillo VA, Kuriyama R, McIntosh JR (1992) A plusend-directed motor enzyme that moves antiparallel microtubules in vitro localizes to the interzone of mitotic spindles. Nature 359: 543-547.

Ohba T, Nakamura M, Nishitani H, Nishimoto T (1999) Selforganization of microtubule asters induced in Xenopus egg extracts by GTP-bound Ran. Science 284: 1356-1358.

Pearson NJ, Cullen CF, Dzhindzhev NS, Ohkura H (2005) A preanaphase role for a $\mathrm{Cks} / \mathrm{Suc1}$ in acentrosomal spindle formation of Drosophila female meiosis. EMBO Rep 6: 1058-1063.

Polanski Z, Ledan E, Brunet S et al. (1998) Cyclin synthesis controls the progression of meiotic maturation in mouse oocytes. Development 125: 4989-4997.

Ribbeck K, Mitchison TJ (2006) Meiotic spindle: sculpted by severing. Curr Biol 16: R923-925.

Riparbelli MG, Callaini G (2005) The meiotic spindle of the Drosophila oocyte: the role of centrosomin and the central aster. J Cell Sci 118: 2827-2836.

Riparbelli MG, Callaini G, Glover DM, Avides Mdo C (2002) A requirement for the abnormal spindle protein to organise microtubules of the central spindle for cytokinesis in Drosophila. J Cell Sci 115: 913-922.

Rutherford SL, Carpenter ATC (1988) The effect of sequence homozygosity on the frequency of X- chromosomal exchange in Drosophila melanogaster females. Genetics 120: $725-732$.

Salmon ED, Begg DA (1980) Functional implications of coldstable microtubules in kinetochore fibers of insect spermatocytes during anaphase. J Cell Biol 85: 853-865.

Segbert C, Barkus R, Powers J, Strome S, Saxton WM, Bossinger O (2003) KLP-18, a Klp2 kinesin, is required for assembly of acentrosomal meiotic spindles in Caenorhabditis elegans. Mol Biol Cell 14: 4458-4469.

Skold HN, Komma DJ, Endow SA (2005) Assembly pathway of the anastral Drosophila oocyte meiosis I spindle. J Cell Sci 118: 1745-1755.

Srayko M, O'Toole ET, Hyman AA, Muller-Reichert T (2006) Katanin disrupts the microtubule lattice and increases polymer number in C. elegans meiosis. Curr Biol 16: 1944-1949.

Steinhauer J, Kalderon D (2006) Microtubule polarity and axis formation in the Drosophila oocyte. Dev Dyn 235: 14551468.

Swan A, Schupbach T (2005) Drosophila female meiosis and embryonic syncytial mitosis use specialized Cks and CDC20 proteins for cyclin destruction. Cell Cycle 4: 1332-1334.

Szollosi D, Calarco P, Donahue RP (1972) Absence of centrioles in the first and second meiotic spindles of mouse oocytes. $J$ Cell Sci 11: 521-541.

Tavosanis G, Llamazares S, Goulielmos G, Gonzalez C (1997) Essential role for gamma-tubulin in the acentriolar female meiotic spindle of Drosophila. EMBO J 16: 1809-1819.

Theurkauf WE, Hawley RS (1992) Meiotic spindle assembly in Drosophila females: behavior of nonexchange chromosomes 
and the effects of mutations in the nod kinesin-like protein. J Cell Biol 116: 1167-1180.

Theurkauf WE, Smiley S, Wong ML, Alberts BM (1992) Reorganization of the cytoskeleton during Drosophila oogenesis: implications for axis specification and intercellular transport. Development 115: 923-936.

Tsurumi C, Hoffmann S, Geley S, Graeser R, Polanski Z (2004) The spindle assembly checkpoint is not essential for CSF arrest of mouse oocytes. J Cell Biol 167: 1037-1050.

Vernos I, Raats J, Hirano T, Heasman J, Karsenti E, Wylie C (1995) Xklp1, a chromosomal Xenopus kinesin-like protein essential for spindle organization and chromosome positioning. Cell 81: 117-127.

Wadsworth P, Khodjakov A (2004) E pluribus unum: towards a universal mechanism for spindle assembly. Trends Cell Biol 14: 413-419.
Wakefield JG, Bonaccorsi S, Gatti M (2001) The Drosophila protein asp is involved in microtubule organization during spindle formation and cytokinesis. J Cell Biol 153: 637-648.

Walczak CE, Vernos I, Mitchison TJ, Karsenti E, Heald R (1998) A model for the proposed roles of different microtubule-based motor proteins in establishing spindle bipolarity. Curr Biol 8: 903-913.

Wassmann K, Niault T, Maro B (2003) Metaphase I arrest upon activation of the Mad2-dependent spindle checkpoint in mouse oocytes. Curr Biol 13: 1596-1608.

Woods LM, Hodges CA, Baart E, Baker SM, Liskay M, Hunt PA (1999) Chromosomal influence on meiotic spindle assembly: abnormal meiosis I in female Mlh1 mutant mice. J Cell Biol 145: $1395-1406$.

Xiang Y, Hawley RS (2006) The mechanism of secondary nondisjunction in Drosophila melanogaster females. Genetics 174: 67-78. 